Chirurg 2020 $91: 780$

https://doi.org/10.1007/s00104-020-01253-0

Online publiziert: 29. Juli 2020

(c) Springer Medizin Verlag GmbH, ein Teil von Springer Nature 2020

O. Strobel · M. W. Büchler

Allgemeinchirurgie, Universitätsklinikum Heidelberg, Heidelberg, Deutschland

\title{
Ein- oder zweizeitige Resektion bei kolorektalem Karzinom mit resektablen synchronen Lebermetastasen
}

operative Technik (offen oder laparoskopisch, Techniken der Anastomosierung und Leberresektion etc.) und Chemotherapie lag bei den Zentren. Bei zweizeitiger Resektion waren die Resektion des Primarius im ersten Eingriff und die Resektion der LM nach einem Intervall von 12 bis 14 Wochen vorgesehen. Primärer Endpunkt war die Rate an MajorKomplikationen innerhalb von 60 Tagen.

tergrund. Bei $15 \%$ der Patienten kolorektalem Karzinomen liegen bei Diagnosestellung Lebermetastasen (LM) vor, bei etwa $10 \%$ primär resektable synchrone LM ohne weitere Fernmetastasen. $\mathrm{Ob}$ bei diesen Patienten eine einzeitige Resektion von Primarius und LM oder eine zweizeitige Resektion erfolgen sollte, ist unklar. Eine einzeitige Resektion könnte mit einem erhöhten Komplikationsrisiko verbunden sein, eine zweizeitige Strategie könnte zu Tumorprogress und sekundärer Irresektabilität führen. $\mathrm{Zu}$ diesem wichtigen Thema lagen bisher nur retrospektive Studien und darauf aufbauende Metaanalysen mit widersprüchlichen Ergebnissen vor.

Methoden. In der multizentrischen randomisiert kontrollierten METASYNCStudie wurden an 10 Zentren in Frankreich Patienten mit kolorektalen Karzinomen und resektablen synchronen LM (Möglichkeit der einzeitigen Resektion ohne Maßnahmen zur Hypertrophieinduktion, keine Notwendigkeit der Gefäßoder Gallenwegsrekonstruktion) und fehlendem Anhalt für extrahepatische Fernmetastasen in die Gruppen einzeitige Resektion und zweizeitige Resektion randomisiert. Die Entscheidung über
Ergebnisse. Von 2006 bis 2015 wurden 105 Patienten randomisiert. In einer modifizierten Intention-to-treat-Analyse wurden 39 Patienten in der einzeitigen und 49 Patienten in der zweizeitigen Gruppe analysiert. Die Gruppen waren prinzipiell hinsichtlich relevanter Einflussparameter vergleichbar bei einem Trend zu mehr Rektumresektionen (31\% vs. $46 \%$ ) und mehr Major-Leberresektionen ( $\geq 3$ Segmente; $31 \%$ vs. $42 \%$ ) in der zweitzeitigen Gruppe. In der zweizeitigen Gruppe wurden die Leberresektionen im Median 17 Wochen nach der Resektion des Primarius durchgeführt. Bei 8 Patienten (17\%) konnte die geplante Leberresektion nicht durchgeführt werden, bei 6 Patienten wegen Progress. Die Rate an Major-Komplikationen war vergleichbar (49\% vs. $46 \%$ ). Es gab einen Trend zu mehr gastrointestinalen Komplikationen in der einzeitigen Gruppe (einzeitig: $28 \%$ vs. zweizeitig: $13 \%$; $p=0,08)$. Die Rate an leberassoziierten Komplikationen war vergleichbar ( $15 \%$ vs. $17 \%)$. Die 2-Jahres-Gesamtüberlebensrate war tendenziell höher nach einzeitiger Resektion ( $87 \%$ vs. $70 \%$; $p=0,05$ : medianes Gesamtüberleben 5,9 vs. 3,9 Jahre).

\section{Kommentar}

Die Autoren folgern, dass eine Strategie der einzeitigen im Vergleich zur zweizeitigen Resektion von Primarius und LM nicht mit einer höheren Komplikationsrate verbunden ist. Eine zweizeitige Strategie könnte mit einem schlechteren Überleben durch Tumorprogression im Intervall zwischen den Eingriffen verbunden sein.

Die Studie hat mehrere wichtige Limitationen. Beilangsamer Rekrutierung haben sich die Therapiestandards der Chirurgie und Chemotherapie seit Design der Studie weiterentwickelt. Eine „Liver-first“-Strategie oder ein kurzes Intervall zwischen den Operationen könnten das Problem der Progression der LM bei zweizeitigem Ansatz lösen. Effektivere prä- und perioperative Chemotherapien beeinflussen onkologische Ergebnisse und Komplikationsraten. Anhand der Studie kann keine Aussage über die beste Strategie bei Konstellationen mit hohem Komplikationsrisiko (z. B. tiefe Rektumresektion und Major-Hepatektomie) getroffen werden.

\section{Korrespondenzadresse}

\section{Strobel}

Allgemeinchirurgie, Universitätsklinikum

Heidelberg

Im Neuenheimer Feld 110, 69120 Heidelberg, Deutschland

Oliver.Strobel@med.uni-heidelberg.de

Interessenkonflikt. O. Strobel und M.W. Büchler geben an, dass kein Interessenkonflikt besteht. 